\title{
Machine learning and artificial neural networks for improved algorithmic design of nanophotonic structures
}

\author{
Didulani Acharige ${ }^{\dagger, *}$, Eric Johlin ${ }^{\dagger}$ \\ † Western University, London, Ontario, N6A 3K7, Canada
}

\section{Introduction}

Nanophotonics is the science and engineering of light-matter interactions that occur at wavelength or subwavelength scales, where the interactions are controlled by the natural or artificial nanostructured matter, as well as their chemical and physical properties. Over the past two decades, nanophotonics has become a promising and active research area, sparked by the growing interest in discovering new physics and technologies with light at the nanoscale. It involves both fundamental studies and numerous applications[1] as modern nanophotonic devices have used complex structures to facilitate innovative applications in optics[2], sensing, computational metamaterials[3], and integrated photonics in a compact and energy-efficient form. With the increase of demands and complexity of nanophotonic devices, the design and optimization of nanophotonic devices become increasingly computationally expensive[4] with numerical simulations due to the immense number of possible combinations of features. As an example, to compute the electromagnetic field profile via Finite-Difference Time-Domain (FDTD)[5] methods require a long simulation time, from several minutes to hours depending on the situation. For design optimization, where it is expected to perform hundreds or even thousands of FDTD simulations, these time requirements can make the process prohibitively expensive. In particular, inverse design, which relies on the computer fully specifying the topology of a new structure, compounds this computational expense, especially if 3D or re-configurable geometries are desired.

Currently, inverse design problems can be solved by using several techniques, with the most common being genetic algorithms and adjoint methods[6]. While having their own strengths and weaknesses, both are computationally expensive and non-trivial to implement, and neither can ensure a globally optimal solution. However, recent theoretical results show that machine learning (ML) and artificial neural network (ANN) techniques are capable of modeling nanophotonic structures for nanophotonic devices, at orders of magnitude lower time per result[7-11].Specifically, neural networks have been used for both forward design[12-15] and inverse design[7, 16-22] of nanophotonic structures.

Furthermore, there is evidence throughout the machine learning literature that the best performance is often achieved by combining vastly different types of approaches. This allows the strength of the one approach to balance the weakness of the other. Even though different studies have looked at few different types of machine learning approaches separately, combining machine learning techniques to achieve a specific task has not yet been explored for optics-related device designs, to the best of our knowledge at this point. Therefore, there is potential for significant improvements through combining more traditional machine learning approaches with ANNs.

Finally, in order to work effectively as an inverse design process, ML and ANN techniques would need to be able to create designs with properties outside of the configuration space in which they have been trained. There is however disagreement and uncertainty about the ability of both interpolation and extrapolation of nanophotonic responses of these machine

*dsalwath@uwo.ca

This article is (C) 2021 by author(s) as listed above. The article is licensed under a Creative Commons Attribution (CC BY 4.0) International license (https://creativecommons.org/licenses/by/4.0/legalcode), except where otherwise indicated with respect to particular material included in the article. The article should be attributed to the author(s) identified above. 
learning processes. While some have claimed such systems cannot extrapolate[23], others have suggested that this might be possible[24]. Therefore, this is still an open area of discussion and literature we are interested to further explore.

\section{Research Plan}

The main objective of the project is to investigate ways to utilize machine learning and artificial neural networks to improve the algorithmic design of nanophotonic structures. As an initial data set, we begin by generating an ensemble of random 2D nanophotonic structures with different optical absorption spectra to train and test neural network architectures. These will be created directly from FDTD simulations. This represents a type of data set that has a property that is relativity simple to check (requires one simulation) but quite difficult to design (requires many simulations).

The first goal of the project is to implement and train a forward network to be able to perform a regression on the created $2 \mathrm{D}$ structures to predict their spectral absorption enhancement. We plan to compare different techniques to find out the best performing algorithm with proper hyperparameter tuning, which is currently lacking in the literature to best of our knowledge at this point. To determine the absorption spectra from the structure image data, we compare the performance of purely deep neural networks (DNN), convolutional neural networks $(\mathrm{CNN})$, as well as their combination with more classical ML dimensionality reduction algorithms to reduce the feature space before feeding to the deep network. The proposed dimensionality reduction approach in this task is the Principal Component Analysis (PCA), although others are being explored as well.

After obtaining the best performing algorithm and architecture for the forward network, the next objective is to implement the inverse design process to determine the best performing system for structure generation. As a first method, a reverse deep neural network with a combination of deep layers and deconvolutional layers is proposed to create new structures from a target spectrum. Next, a conditional generative adversarial network (CGAN)[25] will be tested, and finally, a combination of a reverse network with an autoencoder network will be implemented.

In order to validate the performance and usefulness of the implemented generative architectures, we will begin by testing on spectra purely from the simulation-based validation set. This should indicate if the networks can generate structures that we know are possible, and have been previously introduced into the forward configuration. Subsequently, we will progress to requesting the systems generate structures with spectra outside of the phase space of the training and validation sets. The generated structures can then be verified in FDTD simulations to check the actual response. This should allow us to determine if the structures are indeed able to extrapolate a response outside of the configuration space in which they were trained, as well as map out how far this can be taken.

Our future plans for this study are to design new functional nanophotonic structures for new optoelectronic applications. This includes areas such as improving light and charge collection in photovoltaics, improving optical sensor designs for imaging, and improving the efficiency of single-photon collection for quantum computing.

\section{Acknowledgements}

This work is financially supported by the Natural Sciences and Engineering Research Council of Canada (NSERC) and by Mitacs through the Mitacs Research Training Award. Canadian AI is sponsored by the Canadian Artificial Intelligence Association(CAIAC)[26]. 


\section{References}

[1] A. F. Koenderink and A. Polman. "Nanophotonics: Shrinking light-based technology". In: 348.6234 (2015).

[2] M. H. Tahersima, K. Kojima, T. Koike-Akino, D. Jha, B. Wang, C. Lin, and K. Parsons. "Deep Neural Network Inverse Design of Integrated Nanophotonic Devices". In: Mmi (2018), pp. 1-8. arXiv: 1809.03555. URL: http://arxiv.org/abs/1809.03555.

[3] W. Ma, F. Cheng, and Y. Liu. "Deep-Learning-Enabled On-Demand Design of Chiral Metamaterials". In: ACS Nano 12.6 (2018), pp. 6326-6334. ISSN: 1936086X. DOI: 10.1021/acsnano. 8b03569.

[4] K. Yao, R. Unni, and Y. Zheng. "Intelligent nanophotonics: Merging photonics and artificial intelligence at the nanoscale". In: Nanophotonics 8.3 (2019), pp. 339-366. ISSN: 21928614. DOI: $10.1515 /$ nanoph-2018-0183. arXiv: 1810.11709.

[5] B. Gallinet, J. Butet, and O. J. Martin. "Numerical methods for nanophotonics: Standard problems and future challenges". In: Laser and Photonics Reviews 9.6 (2015), pp. 577-603. ISSN: 18638899. DOI: 10.1002/lpor.201500122.

[6] J. S. Jensen and O. Sigmund. "Topology optimization for nano-photonics". In: Laser and Photonics Reviews 5.2 (2011), pp. 308-321. ISSN: 18638880. DOI: 10.1002/lpor. 201000014.

[7] S. So, T. Badloe, J. Noh, J. Rho, J. Rho, and J. Bravo-Abad. "Deep learning enabled inverse design in nanophotonics". In: Nanophotonics 9.5 (2020), pp. 1041-1057. ISSN: 21928614. DOI: 10.1515/nanoph-2019-0474.

[8] C. C. Nadell, B. Huang, J. M. Malof, and W. J. Padilla. "Deep learning for accelerated alldielectric metasurface design". In: Optics Express 27.20 (2019), p. 27523. ISSN: 1094-4087. DOI: $10.1364 /$ oe 27.027523 .

[9] I. Sajedian, T. Badloe, and J. Rho. "Finding the best design parameters for optical nanostructures using reinforcement learning". In: Arxiv preprint (2018), pp. 1-14. arXiv: 1810.10964. URL: http://arxiv.org/abs/1810.10964.

[10] J. Baxter, A. Calà Lesina, J. M. Guay, A. Weck, P. Berini, and L. Ramunno. "Plasmonic colours predicted by deep learning". In: Scientific Reports 9.1 (2019). ISSN: 20452322. DOI: 10.1038/s41598-019-44522-7. arXiv: 1902.05898.

[11] I. Sajedian, T. Badloe, and J. Rho. "Optimisation of colour generation from dielectric nanostructures using reinforcement learning". In: Optics Express 27.4 (2019), p. 5874. ISSN: 10944087. DOI: $10.1364 /$ oe.27.005874.

[12] D. Gostimirovic and W. N. Ye. "Automating Photonic Design with Machine Learning". In: IEEE International Conference on Group IV Photonics GFP 2018-Augus (2018), pp. 71-72. ISSN: 19492081. DOI: 10.1109/GROUP4.2018.8478722.

[13] Y. Li, Y. Xu, M. Jiang, B. Li, T. Han, C. Chi, F. Lin, B. Shen, X. Zhu, L. Lai, and Z. Fang. "Self-Learning Perfect Optical Chirality via a Deep Neural Network". In: Physical Review Letters 123.21 (2019), pp. 1-6. ISSN: 10797114. DOI: 10.1103/PhysRevLett.123.213902.

[14] T. Zhang, J. Wang, Q. Liu, J. Zhou, J. Dai, X. Han, Y. Zhou, and K. Xu. "Efficient spectrum prediction and inverse design for plasmonic waveguide systems based on artificial neural networks". In: arXiv 7.3 (2018), pp. 368-380. ISSN: 23318422. DOI: 10.1364/prj.7.000368. arXiv: 1805.06410 .

[15] M. Mrejen, I. Malkiel, A. Nagler, U. Arieli, L. Wolf, and H. Suchowski. "Deep Learning for Design and Retrieval of Plasmonic Nanostructures". In: 2019 Conference on Lasers and Electro-Optics (CLEO). 2019, pp. 1-2. DOI: 10.1364/CLEO_QELS.2019.FTu4C.3.

[16] K. Kojima, Y. Tang, T. Koike-Akino, Y. Wang, D. Jha, K. Parsons, M. H. Tahersima, F. Sang, J. Klamkin, and M. Qi. "Inverse Design of Nanophotonic Devices using Deep Neural Networks". In: Asia Communications and Photonics Conference (2020). URL: https://www. merl.com.

[17] R. S. Hegde. "Deep learning: a new tool for photonic nanostructure design". In: Nanoscale Advances 2.3 (2020), pp. 1007-1023. ISSN: 25160230. DOI: 10.1039/c9na00656g.

[18] J. Jiang and J. A. Fan. "Global Optimization of Dielectric Metasurfaces Using a PhysicsDriven Neural Network". In: Nano Letters 19.8 (2019), pp. 5366-5372. ISSN: 15306992. DOI: 10.1021/acs.nanolett.9b01857. arXiv: 1906.04157. 
[19] I. Malkiel, M. Mrejen, A. Nagler, U. Arieli, L. Wolf, and H. Suchowski. "Plasmonic nanostructure design and characterization via Deep Learning". In: Light: Science and Applications 7.1 (2018). ISSN: 20477538. DOI: 10.1038/s41377-018-0060-7. URL: http://dx.doi.org/ 10.1038/s41377-018-0060-7.

[20] Y. Chen, J. Zhu, Y. Xie, N. Feng, and Q. H. Liu. "Smart inverse design of graphene-based photonic metamaterials by an adaptive artificial neural network". In: Nanoscale 11.19 (2019), pp. 9749-9755. ISSN: 20403372. DOI: 10.1039/c9nr01315f.

[21] M. H. Tahersima, K. Kojima, T. Koike-Akino, D. Jha, B. Wang, C. Lin, and K. Parsons. "Deep Neural Network Inverse Design of Integrated Photonic Power Splitters". In: Scientific Reports 9.1 (2019), pp. 1-9. ISSN: 20452322. DOI: 10 . 1038/s41598-018-37952-2. URL: http://dx.doi.org/10.1038/s41598-018-37952-2.

[22] D. Liu, Y. Tan, E. Khoram, and Z. Yu. "Training Deep Neural Networks for the Inverse Design of Nanophotonic Structures". In: ACS Photonics 5.4 (2018), pp. 1365-1369. ISsN: 23304022. DOI: $10.1021 /$ acsphotonics.7b01377. arXiv: 1710.04724.

[23] A. W. Minns and M. J. Hall. "Modélisation pluie-débit par des réseaux neuroneaux artificiels". In: Hydrological Sciences Journal 41.3 (1996), pp. 399-417. ISSN: 21503435. DOI: $10.1080 /$ 02626669609491511.

[24] J. Jiang, D. Sell, S. Hoyer, J. Hickey, J. Yang, and J. A. Fan. "Free-form diffractive metagrating design based on generative adversarial networks". In: ACS Nano 13.8 (2019), pp. 88728878. ISSN: 1936086X. DOI: 10.1021/acsnano.9b02371.

[25] S. So and J. Rho. "Designing nanophotonic structures using conditional-deep convolutional generative adversarial networks". In: arXiv 8.7 (2019), pp. 1255-1261. ISSN: 23318422.

[26] Canadian Artificial Intelligence Association. 2020. URL: https://www.caiac.ca/. 